\title{
FORTALEZAS Y VIRTUDES PERSONALES, FELICIDAD SUBJETIVA, SENTIDO DEL HUMOR Y PERSONALIDAD. ANÁLISIS SEGÚN EL PAÍS Y GÉNERO DE LOS DOCENTES
}

\author{
Lucía Herrera Torres \\ Facultad de Ciencias de la Educación y del Deporte de Melilla \\ Departamento de Psicología Evolutiva y de la Educación \\ Universidad de Granada, España \\ luciaht@ugr.es \\ Teresa María Perandones González \\ Facultad de Ciencias de la Educación y del Deporte de Melilla \\ Departamento de Psicología Evolutiva y de la Educación \\ Universidad de Granada, España \\ tmperandones@ugr.es
}

Recepción Artículo: 6 septiembre 2021

Admisión Evaluación: 6 septiembre 2021

Informe Evaluador 1: 18 septiembre 2021

Informe Evaluador 2: 23 septiembre 2021

Aprobación Publicación: 09 septiembre 2021

\section{RESUMEN}

Las variables personales de los docentes, desde la perspectiva de la Psicología Positiva, son de especial relevancia en el ámbito educativo. Al respecto, este trabajo tiene como objetivo principal analizar las fortalezas y virtudes personales, felicidad subjetiva, sentido del humor y personalidad de los docentes en función del país de origen (España y República Dominicana) y el género. Formaron parte del estudio 454 docentes de las diferentes etapas educativas, 76.9 \% de España y 23.1\% de República Dominicana. En función del género, 156 eran hombres (34.4 \%) y 298 mujeres (65.6 \%). La edad mínima fue de 19 y la máxima de 66 (Edad media = 40.04 años, DT $=10.51)$. Para la recogida de información se emplearon los siguientes instrumentos: Valores en Acción Inventario de Fortalezas (VIA-IS), Escala de Felicidad Subjetiva (SHS), Escala Multidimensional del Sentido del Humor (MSHS) e Inventario de Personalidad de los Cinco Factores (BFl-10). Se hallaron diferencias en función del país y el género. Al respecto, los docentes de República Dominicana puntuaron más alto que los de España en fortalezas y virtudes personales, felicidad subjetiva, sentido del humor y en el factor de personalidad Apertura a nuevas experiencias. Los docentes españoles, por su parte, puntuaron más alto en el factor de personalidad Neuroticismo. En función del género, las mujeres alcanzaron valores más altos en la escala Humanidad del VIAIS, en la felicidad subjetiva y en el factor de personalidad Extroversión. Se discute la relevancia del estudio de los constructos psicológicos analizados en los docentes, dado que implican procesos cognitivos, afectivos y motivacionales conducentes a un desempeño exitoso. Igualmente, las diferencias culturales entre los dos países de comparación y en función del género. Se estable la necesidad de llevar a cabo estudios transculturales, así como profundizar en los estudios de género. 


\section{FORTALEZAS Y VIRTUDES PERSONALES, FELICIDAD SUBJETIVA, SENTIDO DEL HUMOR Y PERSONALIDAD.}

ANÁLISIS SEGÚN EL PAISS Y GENERO DE LOS DOCENTES

Palabras clave: psicología positiva; variables personales; docentes; país; género.

\section{ABSTRACT}

Personal strengths and virtues, subjective happiness, sense of humor and personality. Analysis by country and gender of teachers. The personal variables of teachers, from the perspective of Positive Psychology, are of special relevance in the educational field. In this regard, the main objective of this work is to analyze the strengths and personal virtues, subjective happiness, sense of humor and personality of teachers according to the country of origin (Spain and the Dominican Republic) and gender. 454 teachers from different educational stages took part in the study, 76.9\% from Spain and 23.1\% from the Dominican Republic. Based on gender, 156 were men (34.4\%) and 298 women (65.6\%). The minimum age was 19 and the maximum was 66 (Mean age $=40.04$ years, $S D=10.51$ ). The following instruments were used for data collection: Values in Action Inventory of Strengths (VIA-IS), Subjective Happiness Scale (SHS), Multidimensional Sense of Humor Scale (MSHS) and Big Five Inventory (BFI -10). Differences were found according to country and gender. In this regard, teachers in the Dominican Republic scored higher than those in Spain in personal strengths and virtues, subjective happiness, sense of humor and in the personality factor Openness to new experiences. Spanish teachers, for their part, scored higher in the Neuroticism personality factor. Based on gender, women achieved higher values on the VIA-IS Humanity scale, in subjective happiness and in the Extroversion personality factor. The relevance of the study of the psychological constructs analyzed in teachers is discussed, given that they involve cognitive, affective and motivational processes leading to successful performance. Likewise, the cultural differences between the two comparison countries and based on gender. The need to carry out cross-cultural studies is established, as well as to deepen in gender studies.

Keywords: positive psychology; personal variables; teachers; country; gender.

\section{INTRODUCCIÓN}

Las variables personales de los docentes, desde la perspectiva de la Psicología Positiva (Seligman \& Csikszentmihalyi, 2000), son de especial relevancia en el ámbito educativo (Seligman et al., 2009; Yin \& Majid, 2018). Un buen profesional de la enseñanza debe ser consciente de sus propias emociones y controlarlas, tener la habilidad de automotivarse, empatizar con los demás y disponer de las habilidades sociales necesarias tanto para crear y mantener relaciones positivas como para reconocer los conflictos y afrontarlos positivamente (Torrijos et al., 2018). En este sentido, ha de desarrollar determinadas habilidades de introspección y reconocimiento de sus puntos fuertes y débiles, poseer confianza en sí mismo, así como prestar atención a su estado de ánimo y la expresión que haga del mismo ante el alumnado (Herman et al., 2018).

Así, las fortalezas hacen referencia a las cualidades del ser humano que potencian una vida más plena y positiva, aportando las estrategias psicológicas necesarias para ello (Ihsan \& Furnham, 2018). En esta dirección, Peterson y Seligman (2000) establecen 24 fortalezas personales que se engloban en 6 virtudes humanas: Sabiduría y Conocimiento, Coraje, Humanidad y Amor, Justicia, Contención, y Trascendencia. Igualmente, dentro de la Psicología Positiva se encuadra el constructo de felicidad subjetiva (Seligman \& Csikszentmihalyi, 2000), denominado también bienestar psicológico (Ryff, 1989, 1995). Al respecto, docentes con afectos positivos informan de un mayor empleo de estrategias de enseñanza efectivas (Moè et al., 2010).

Sumado a lo anterior, el humor tiene un enorme valor como evocador de emociones positivas y, por lo tanto, de los beneficios asociados a ellas. El humor proporciona, asimismo, un bienestar emocional a corto plazo (A çam, 2017). El uso del sentido del humor en el ámbito educativo posibilita la eficacia comunicativa y convierte el mensaje en más persuasivo, generando ánimo positivo y atracción interpersonal (Martin, 2008).

Por su parte, el Five Factor Model (McCrae \& Costa, 1997) es el modelo de personalidad que más desarrollo ha tenido en la literatura científica (Herrera et al., 2020). Este modelo postula que los cinco grandes factores de la personalidad (Extraversión, Amabilidad, Responsabilidad, Neuroticismo, Apertura) se encuentran en el nivel más alto de la jerarquía de la personalidad. La personalidad de los docentes, evaluada a través de este modelo, predice la eficacia docente percibida (Kim et al., 2018). 


\section{OBJETIVOS DE LA INVESTIGACIÓN}

En función de lo descrito, el objetivo principal de este trabajo es analizar las fortalezas y virtudes personales, felicidad subjetiva, sentido del humor y personalidad de los docentes en función del país de origen (España y República Dominicana) y el género.

\section{PARTICIPANTES}

El tipo de muestreo empleado para la selección de la muestra fue de tipo no probabilístico por conveniencia. Al respecto, en el estudio participaron 454 docentes de las diferentes etapas educativas (desde educación infantil hasta la educación superior), $76.9 \%$ de España ( $n=349$ ) y 23.1\% de República Dominicana $(n=105)$. En función del género, el $34.4 \%$ eran hombres $(n=156)$ y el $65.6 \%$ mujeres $(n=298)$.

\section{INSTRUMENTOS}

Los cuatro instrumentos empleados para la recogida de información se describen a continuación.

Valores en Acción Inventario de Fortalezas (VIA-IS)

El Values in Action Inventory of Strengths (VIA-IS; Peterson \& Seligman, 2000), está formado por 24 ítems a los que se ha de contestar según una escala tipo Likert de 5 puntos. Evalúa la frecuencia con la que el individuo se percibe realizando conductas representativas de las 24 fortalezas y 6 virtudes analizadas (Sabiduría y Conocimiento, Coraje, Humanidad y Amor, Justicia, Contención, y Trascendencia).

La fiabilidad del instrumento en esta investigación, medida mediante el índice de consistencia interna Alfa de Cronbach, fue de $\alpha=.894$. En cada una de las escalas del cuestionario se obtuvieron los siguientes valores: Sabiduría y Conocimiento, $\alpha=.785$, Coraje, $\alpha=.721$, Humanidad y Amor, $\alpha=.729$, Justicia, $\alpha=.659$, Contención, $\alpha=.703$, y Trascendencia, $\alpha=.628$.

Escala de Felicidad Subjetiva (SHS)

Se tradujo al español el Subjective Happiness Scale (SHS; Lyubomirsky \& Lepper, 1999). Esta prueba la componen 4 ítems a los que responde atendiendo a una escala tipo Likert de 7 puntos ( $1=$ Menos feliz; $7=$ Más feliz; $1=$ Nada en absoluto; $7=$ Muchísimo). Mide la felicidad subjetiva global mediante enunciados en los que la persona se evalúa a sí misma o bien se compara con quienes le rodean.

La fiabilidad hallada en este estudio es de $\alpha=.70$, similar a la hallada por Extremera et al. (2005), donde también se utilizó una adaptación al español de este instrumento y la fiabilidad fue de $\alpha=.72$.

Escala Multidimensional del Sentido del Humor (MSHS)

El Multidimensional Humor Sense Scale (MSHS; Thorson \& Powell, 1991) está formado por 24 ítems a los que se ha de responder siguiendo una escala tipo Likert de cinco puntos ( 1 = Muy en desacuerdo, $5=$ Muy de acuerdo). Dichos ítems conforman tres factores: Factor I. Competencia o habilidad personal para utilizar el humor, Factor II. El humor como mecanismo de control de la situación y Factor III. Valoración social y actitudes hacia el humor.

La fiabilidad total para esta investigación fue de $\alpha=.925$. Por su parte, en los factores se obtuvieron los siguientes resultados: Factor I, $\alpha=.941$; Factor II, $\alpha=.795$; Factor III, $\alpha=.615$.

Inventario de Personalidad de los Cinco Factores (BFI-10)

Se empeló el Big Five Inventory-10 ítems (BFI-10; Rammstedt \& John, 2007), realizando una traducción propia al español. El índice de consistencia general para este trabajo fue de $\alpha=.419$. Por factores, el estadístico $\alpha$ de Cronbrach indicó los siguientes valores: Extraversión $=.602$, Amabilidad $=.186$, Responsabilidad $=.392$, Neuroticismo $=.600$, Apertura $=.439$. Como se puede observar, los valores son bajos en unos casos y moderados en otros.

\section{PROCEDIMIENTO}

Para el proceso de recogida de información se contó con el consentimiento informado de cada uno de los participantes. Se pasaron los cuatro instrumentos de evaluación de forma conjunta. 
Respecto al procedimiento seguido para el análisis estadístico de datos, se empleó el paquete estadístico SPSS versión 24. Se desarrolló un Análisis de varianza multivariante en el que como variables dependientes se introdujeron los diferentes factores o escalas de los cuatro instrumentos de evaluación empleados y como variables independientes el país y el género de los participantes. Para las comparaciones post-hoc se empleó el estadístico Bonferroni y para la evaluación del tamaño del efecto Eta-cuadrado parcial.

\section{RESULTADOS}

Los resultados fueron significativos en función de la variable país, $F=7.699, p=.000$, Eta2 $p=.211$, así como el género, $F=2.927, p=.000$, Eta2 $p=.092$, pero no la interacción de ambos factores, $F=.920, p=.541$, Eta2 $p=.031$. En la Tabla 1 se presentan los estadísticos descriptivos así como resultados del análisis de varianza implementado para cada una de las dimensiones de los cuatro instrumentos de evaluación atendiendo a las dos variables de comparación. 
APRENDIENDO EN POSITIVO: SUPERANDO EL COVID

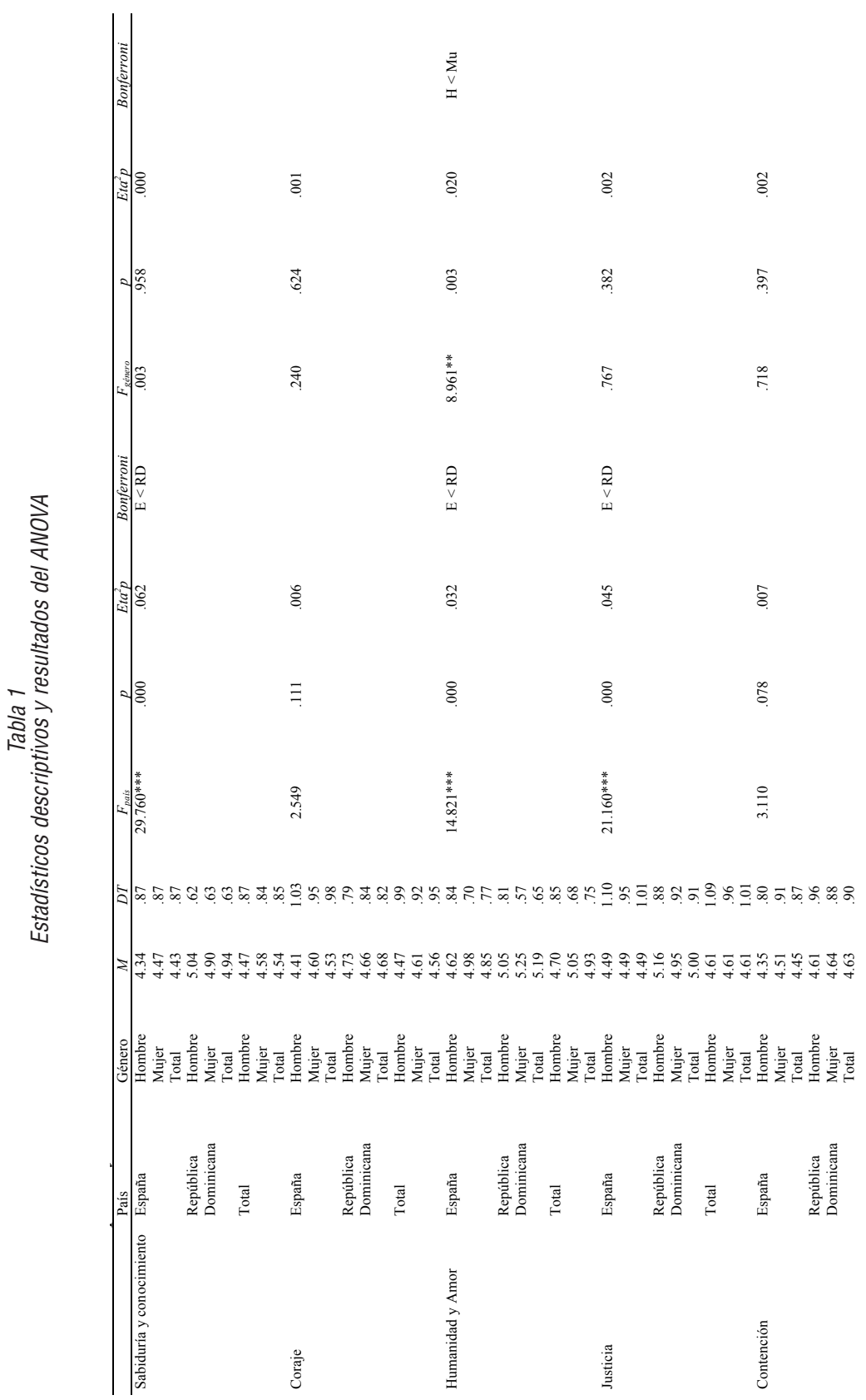


FORTALEZAS Y VIRTUDES PERSONALES, FELICIDAD SUBJETIVA, SENTIDO DEL HUMOR Y PERSONALIDAD. ANÁLISIS SEGÚN EL PAÍS Y GÉNERO DE LOS DOCENTES

$\underset{\substack{\Sigma \\ \Sigma}}{v}$

๕̊.

ธิ

ڤั

ळ.

$\overline{8}$

$\frac{1}{0}$

ํำ

ธิ

$\stackrel{\infty}{5}$

ลุ

in

สี

$\stackrel{\infty}{\sim}$

旁

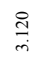

$\stackrel{5}{5}$

m

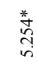

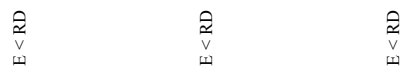

$\stackrel{n}{\varrho}$

ััธ

g̣

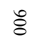

§

ธิ

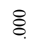

ธิ

$\S$

gे

হ

ले

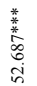

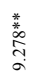

तै

$\stackrel{\text { i }}{\stackrel{i}{i}}$

5

$\frac{\infty}{\sigma}$

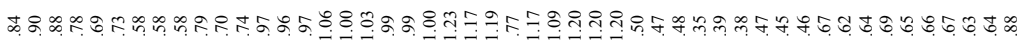

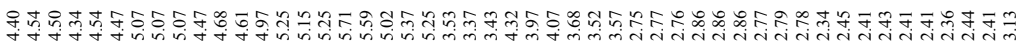

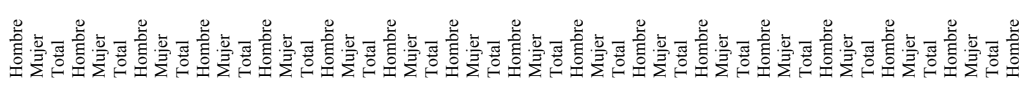

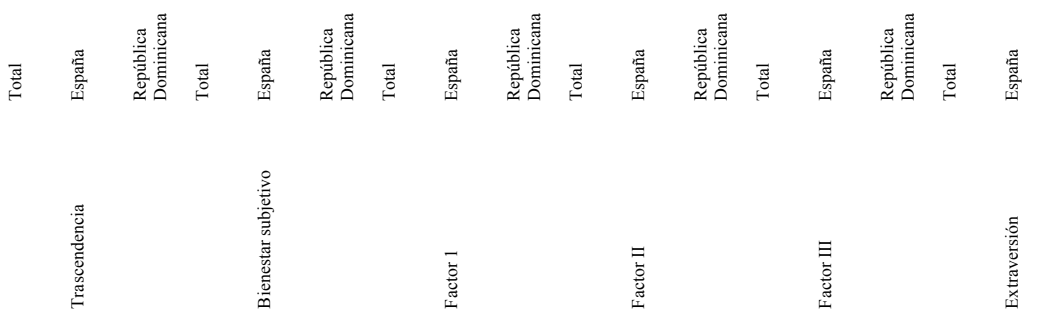


APRENDIENDO EN POSITIVO: SUPERANDO EL COVID

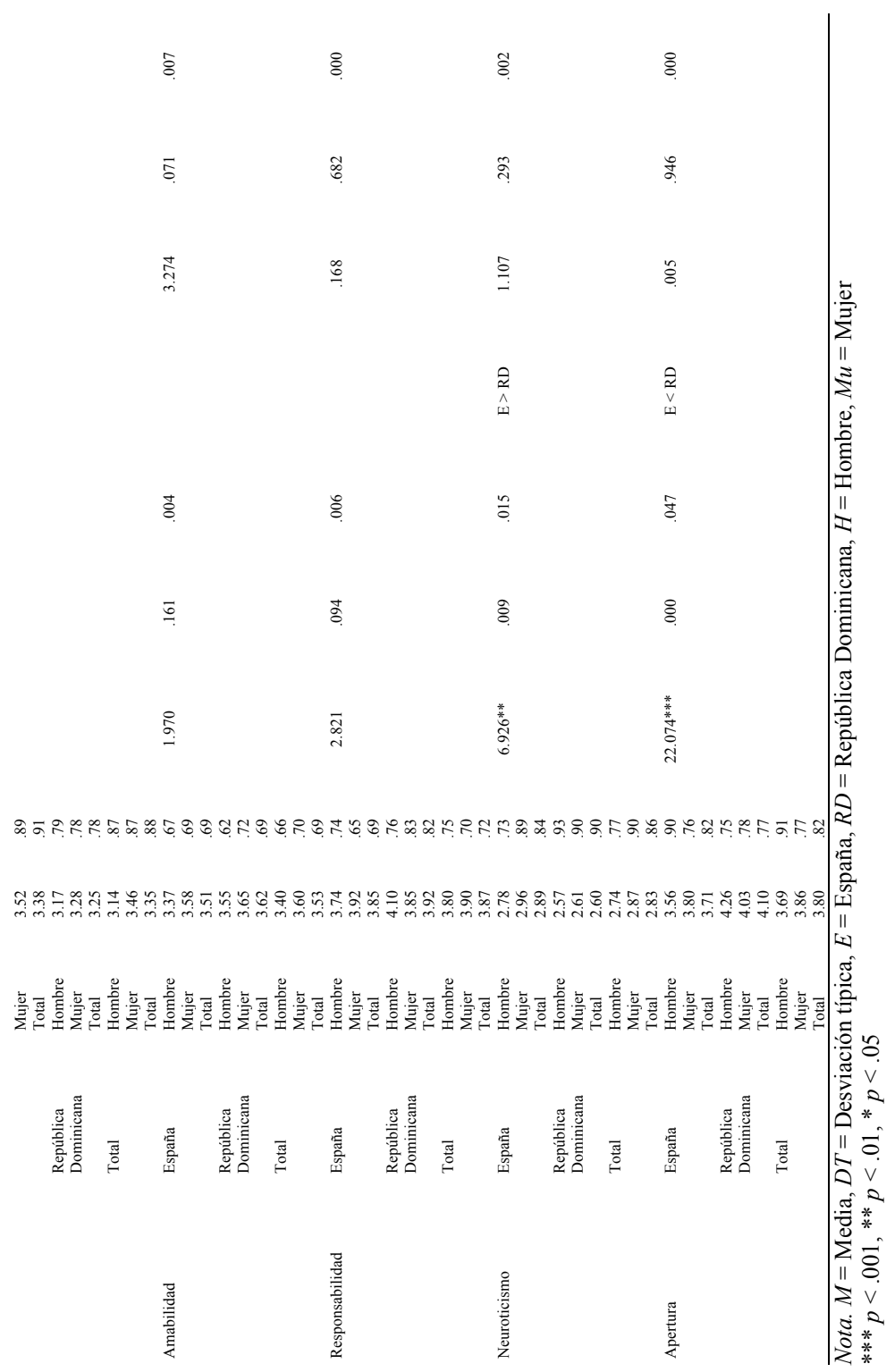




\section{FORTALEZAS Y VIRTUDES PERSONALES, FELICIDAD SUBJETIVA, SENTIDO DEL HUMOR Y PERSONALIDAD. ANÁLISIS SEGÚN EL PAIIS Y GÉNERO DE LOS DOCENTES}

\section{DISCUSIÓN}

En función de los resultados, los docentes de República Dominicana puntuaron más alto que los de España en las siguientes fortalezas y virtudes personales: Sabiduría y conocimiento, Humanidad y Amor, Justicia y Trascendencia. En el contexto educativo, dichos aspectos están relacionados con la conducta prosocial, el liderazgo, la autoestima, un mayor ajuste psicológico y control del estrés, así como un mejor nivel de realización tanto personal como prosocial (Seligman et al., 2009).

Igualmente, se encontró que los docentes dominicanos alcanzaban puntuaciones más altas en su bienestar subjetivo o felicidad subjetiva, junto con el factor del sentido del humor relativo a Competencia o habilidad personal para utilizar el humor y el factor de personalidad Apertura. Esto puede ser debido a factores culturales puesto que la cultura dominicana se centra más en vivir y valorar el momento de una forma positiva y enfrentarse a las situaciones nuevas con humor y cercanía personal. Sumado a ello, los estudios muestran que las personas con altas puntuaciones en bienestar subjetivo interpretan los acontecimientos vitales y las situaciones diarias de una forma que les ayuda a mantener su nivel de felicidad (Lyubomirsky et al., 2015). Además, el sentido del humor genera a corto plazo más emociones positivas, bienestar emocional y menor ansiedad (Adil et al., 2016; A çam, 2017; Muchmore et al., 2016). Por su parte, la Apertura a nuevas experiencias se relaciona directamente en los docentes con la inteligencia emocional (Alghamdi et al., 2017), el optimismo (Akhtar \& Saleem, 2020), su desempeño docente y el compromiso con el trabajo, además de la puesta en práctica de estilos motivacionales que fomentan la autonomía (Reeve et al., 2018).

Por el contrario, los docentes españoles puntuaron más alto que los dominicanos en el factor de personalidad Neuroticismo, aunque en ambos casos las puntuaciones fueron más bien bajas. La literatura científica pone de manifiesto que el neuroticismo predice negativamente la satisfacción con la vida de los docentes ( $D$ dová et al., 2020).

Atendiendo a la variable género, las mujeres obtuvieron valores más altos que los hombres en la escala Humanidad y Amor del VIA-IS, tal y como se indica en estudios previos (Heintz et al., 2019), en la felicidad subjetiva (Salavera \& Usán, 2021) y en el factor de personalidad Extroversión (Weisberg et al., 2011).

\section{CONCLUSIONES}

Tomando en su conjunto lo descrito hasta el momento, se pone de manifiesto la implicación de procesos cognitivos, afectivos y motivacionales conducentes a un desempeño exitoso, por lo que han de tenerse en cuenta en el desarrollo profesional del docente (Caprara et al., 2010; David et al., 2013; Herman et al., 2018; Kim et al., 2018; Moè et al., 2010; Oishi, 2012; Yin \& Majid, 2018).

Asimismo, las diferencias culturales entre los dos países de comparación revelan la necesidad de llevar a cabo estudios transculturales (Heintz et al., 2019; Kocjan et al., 2021) que, a su vez, atiendan a la variable género.

\section{REFERENCIAS BIBLIOGRÁFICAS}

Adil, A., Ishaq, G., \& Khan, 0. (2016). Impact of Sense of Humor on Perceived Stress among Undergraduate Medical Students: A Gendered Perspective. Journal of Gender \& Social Issues, 15(1), 57-67.

A çam, R. (2017). Investigating instructors' perceptions on the use of humour in higher education. European Journal of Education Studies, 3(2), 238-248. https://doi.org/10.5281/zenodo.259493

Akhtar, N., \& Saleem, S. (2020). Happiness and Personality Traits as Predictors of Optimism in School and College Teachers. Pakistan Journal of Psychological Research, 35(4), 739-751.

https://doi:org/10.33824/PJPR.2020.35.4.40

Alghamdi, N. G., Aslam, M., \& Khan, K. (2017). Personality Traits as Predictor of Emotional Intelligence among the University Teachers as Advisors. Education Research International:9282565.

https://doi.org/10.1155/2017/9282565

Caprara, G. V., Alessandri, G., Di Giunta, L., Panerai, L., \& Eisenberg, N. (2010). The contribution of agreeable- 
ness and self-efficacy beliefs to prosociality. European Journal of Personality, 24(1), 36-55. https://doi.org/10.1002/per.739

David, S. A., Boniwell, I., \& Conley, A. (2013). The Oxford handbook of happiness. Oxford University Press.

D dová, M., Bizová, N., \& Baník, G. (2020). Life satisfaction of teachers from pre-school and elementary education in the context of personality traits, sociodemographic factors, and factors related to school and teaching. Psychology and Education Journal, 57(6), 429-434.

Extremera, N., Durán, A., \& Rey, L. (2005). La inteligencia emocional percibida y su influencia sobre la satisfacción vital, la felicidad subjetiva y el engagement en trabajadores de centros para personas con discapacidad intelectual. Ansiedad y Estrés, 11(1), 63-73.

Heintz, S., Kramm, C., \& Ruch, W. (2019). A meta-analysis of gender differences in character strengths and age, nation, and measure as moderators. The Journal of Positive Psychology, 14(1), 103-112. https://doi.org/10.1080/17439760.2017.1414297

Herman, K. C., Hickmon-Rosa, J. E., \& Reinke, W. M. (2018). Empirically derived profiles of teacher stress, burnout, self-efficacy, and coping and associated student outcomes. Journal of Positive Behavior Interventions, 20(2), 90-100. https://doi.org/10.1177/1098300717732066

Herrera, L., Al-Lal, M., \& Mohamed, L. (2020). Academic Achievement, Self-Concept, Personality and Emotional Intelligence in Primary Education. Analysis by Gender and Cultural Group. Frontiers in Psychology, 10:3075. https://doi.org/10.3389/fpsyg.2019.03075

Ihsan, Z., \& Furnham, A. (2018). The Measurement of Character Strengths and Virtues. In A. Kumar, T. S. George, \& N. T. Sudhesh (Eds.), Character Strength Development: Perspectives from Positive Psychology (pp. 92112). Sage Publications.

Kim, L. E., Dar-Nimrod, I., \& MacCann, C. (2018). Teacher personality and teacher effectiveness in secondary school: Personality predicts teacher support and student self-efficacy but not academic achievement. Journal of Educational Psychology, 110(3), 309-323. https://doi.org/10.1037/edu0000217

Kocjan, G. Z., Jose, P. E., Socan, G., \& Avsec, A. (2021). Measurement Invariance of the Subjective Happiness Scale Across Countries, Gender, Age, and Time. Assessment. Advance online publication. https://doi.org/10.1177/1073191121993558

Lyubomirsky, S., \& Lepper, H. (1999). A measure of subjective happiness: Preliminary reliability and construct validation. Social Indicators Research, 46(2), 137-155. https://doi.org/10.1023/A:1006824100041.

Lyubomirsky, S., Layous, K., Chancellor, J., \& Nelson, S. K. (2015). Thinking about rumination: the scholarly contributions and intellectual legacy of Susan Nolen-Hoeksema. Annual Review of Clinical Psychology, 11, 122. https://doi.org/10.1146/annurev-clinpsy-032814-112733

Martin, R. (2008). La Psicología del humor. Un enfoque integrador. Orión Ediciones.

McCrae, R. R., \& Costa, P. T. (1997). Personality trait structure as a human universal. American Psychologist, 52(5), 509-516. https://doi.org/10.1037/0003-066X.52.5.509

Moè, A., Pazzaglia, F., \& Ronconi, L. (2010). When being able is not enough. The combined value of positive affect and self-efficacy for job satisfaction in teaching. Teaching and Teacher Education, 26, 1145-1153. https://doi.org/10.1016/j.tate.2010.02.010.

Muchmore, J., Griggs, T., Tidwell, D., Mnayer, M., \& Beyer, B. K. (2016). Jokes, puns, and emoticons: the roles and functions of impromptu humor in our teacher education practices. In D. Garbett, \& A. Ovens (Eds.), Enacting self-study as methodology for professional inquiry. Self-Study of Teacher Education Practices (pp. 419-425). University of Auckland.

Oishi, S., Diener, E., \& Lucas, R. E. (2007). The optimum level of well-being: Can people be too happy? Perspectives on Psychological Science, 2(4), 346-360. https://doi.org/10.1007/978-90-481-2350-6_8

Peterson, C., \& Seligman, M. E. P. (2000). Values in action (VIA): Classification of strengths. Values in Action Institute. 
Rammstedt, B., \& John, 0. P. (2007). Measuring personality in one minute or less: A 10-item short version of the Big Five Inventory in English and German. Journal of Research in Personality, 41(1), 203-212. https://doi.org/10.1016/j.jrp.2006.02.001

Reeve, J., Jang, H. R., \& Jang, H. (2018). Personality-based antecedents of teachers' autonomy-supportive and controlling motivating styles. Learning and Individual Differences, 62, $12-22$. https://doi.org/10.1016/j.lindif.2018.01.001

Ryff, C. D. (1989). Happiness is everything, or is it? Explorations on the meaning of psychological well-being. Journal of Personality and Social Psychology, 57(6), 1069-1081. https://doi.org/10.1037/00223514.57.6.1069

Ryff, C. D. (1995). Psychological well-being in adult life. Current Directions in Psychological Science, 4(4), 99104. https://doi.org/10.1111/1467-8721.ep10772395

Salavera, C., \& Usán, P. (2021). Relationship between Social Skills and Happiness: Differences by Gender. International Journal of Environmental Research and Public Health, 18(15): 7929. https://doi.org/10.3390/ijerph18157929

Seligman, M. E. P., \& Csikszentmihalyi, M. (2000). Positive psychology: An introduction. American Psychologist, 55(1), 5-14. https://doi.org/10.1037/0003-066X.55.1.5

Seligman, M. E. P., Ernst, R. M., Gillham, J., Reivich, K., \& Linkins, M. (2009). Positive education: Positive psychology and classroom interventions. Oxford Review of Education, 35(3), 293-311. https://doi.org/10.1080/03054980902934563

Thorson, J. A., \& Powell, F. C. (1991). Measurement of sense of humor. Psychological Reports, 69(2), 691-702. https://doi.org/10.2466/pro.1991.69.2.691

Torrijos, P., Martín, J. F., \& Rodríguez, M. J. (2018). La educación emocional en la formación permanente del profesorado no universitario. Profesorado. Revista de Currículum y Formación del Profesorado, 22(1), 595-613. https://recyt.fecyt.es/index.php/profesorado/article/view/63662

Weisberg, Y. J., DeYoung, C.G., \& Hirsh, J. B. (2011). Gender differences in personality across the ten aspects of the Big Five. Frontiers in Psychology, 2:178. https://doi.org/10.3389/fpsyg.2011.00178

Yin, L. C., \& Majid, R. A. (2018). The Goodness of Character Strengths in Education. International Journal of Academic Research in Business and Social Sciences, 8(6), 1237-1251.

https://doi.org/10.6007/IJARBSS/v8-i6/4512 